\title{
A Study on Polymyxin B Induced Nephrotoxicity: A Systematic Review
}

\author{
Rasika.S.Kumar ${ }^{1 *}$, Anitha. $E^{1}$, Mohd Junaid ${ }^{1}$, Dr. Samhitha Chetty ${ }^{2}$ \\ 1 - Pharm D, K.K. College of Pharmacy, Chennai, Tamilnadu, India. \\ 2 - Assistant Professor, Department of Pharmacy Practice, K.K. College of Pharmacy, Chennai, Tamilnadu, India. \\ *Corresponding author's E-mail: rasika.s.kumar98@gmail.com
}

Received: 02-12-2021; Revised: 26-01-2022; Accepted: 05-02-2022; Published on: 15-02-2022.

\begin{abstract}
Polymyxins are a group of cationic polypeptide antibiotics effective against several Gram-negative bacteria. The emerging experience with Polymyxin B, indicated that up to half of the patients receiving this drug presented nephrotoxicity. More recent studies using standardized criteria for AKI, such as Kidney Disease: Improving Global Outcomes (KDIGO), Acute Kidney Injury Network (AKIN) and Risk, Injury, Failure, Loss of kidney function, and End-stage kidney disease (RIFLE), have been published, among which, a recent metaanalysis showed that the occurrence of AKI remained undesirably high with mean rates of $31.3 \%, 32.6 \%$ and $39.4 \%$, respectively. Polymyxins act primarily upon the external and cytoplasmic membranes, with similar action to that of simple cationic detergents. The mechanism of action is thought to be based on surfactant activity, which disrupts the bacterial outer and cytoplasmic membranes. Polymyxin B is used at the dose of 1.5 to $2.5 \mathrm{mg} / \mathrm{kg} /$ day $(1.0 \mathrm{mg}$ of polymyxin $B=10,000 \mathrm{IU})$ in patients with normal renal function. The development of renal injury, is the major limiting factor for the use of this class of antibiotics. Identifying ways to minimize nephrotoxicity associated with polymyxin B use is critical to advance clinical practice.
\end{abstract}

Keywords: Pentacationic polypeptides, nephrotoxicity, resistance, therapeutic drug monitoring.

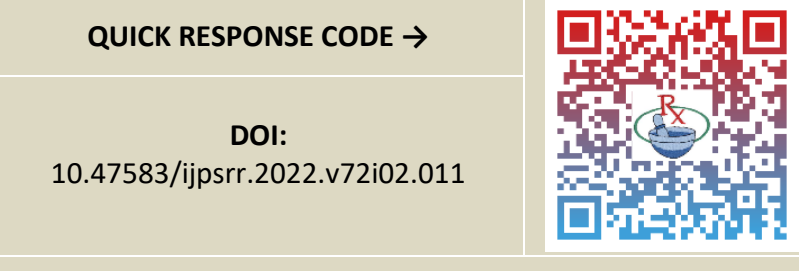

DOI link: http://dx.doi.org/10.47583/ijpsrr.2022.v72i02.011

\section{INTRODUCTION}

$\mathrm{P}$

olymyxins are a group of cationic polypeptide antibiotics. Polymyxin B is a lipopeptide antibiotic isolated from bacillus polymyxa. It differs from colistin in having D-phenylalanine in place of D-leucine. Polymyxins belong to a group of active antibiotics against several Gram-negative bacteria. Synthesized by Bacillus polymyxa strains, they were independently described in 1947 by U.S. and English researchers and the generic name of polymyxins was adopted for the antibiotics derived from this microorganism. They contain five closely related substances, called polymyxins A, B, C, D and E, the latter of which is known as colistin, produced by Bacillus colistinus. Only polymyxins $B$ and $E$ have clinical use, due to the high toxicity of the other polymyxins. ${ }^{1}$

Polymyxin $B$ is a mixture of four Polymyxin components (PB1, PB2, PB3, and PB4) with its major components consisting of Polymyxin B1 (PB1) and Polymyxin B2 (PB2). For clinical use, Polymyxin $B$ is administered intravascularly, intrathecally, aerosolized, or topically as Polymyxin B sulfate. It is not used orally due to poor bioavailability. ${ }^{2}$
Polymyxin $B$ is available for parenteral use as sulphate salt. As reported, the emerging experience with Polymyxin $B$, indicated that up to half of the patients receiving this drug presented nephrotoxicity. However, it became clear that multiple definitions for nephrotoxicity impaired a more accurate estimate of the real incidence of acute kidney injury (AKI) in patients treated with Polymyxins.

More recent studies using standardized criteria for AKI, such as Kidney Disease: Improving Global Outcomes (KDIGO), Acute Kidney Injury Network (AKIN) and Risk, Injury, Failure, Loss of kidney function, and End-stage kidney disease (RIFLE), have been published, among which, a recent meta-analysis showed that the occurrence of AKI remained undesirably high with mean rates of $31.3 \%$, $32.6 \%$ and $39.4 \%$, respectively.

\section{Antimicrobial activity}

The antimicrobial spectrum includes only Gram-negative organisms. An epidemiological surveillance program (SENTRY - 2001 to 2004) found that polymyxin B was excellent against Pseudomonas aeruginosa $(1.3 \%$ of resistance), Acinetobacter spp (2.1\% of resistance), Citrobacter spp., Escherichia coli and Klebsiella spp (less than $2 \%$ of resistance). Polymyxins are not active against Gram-positive bacteria and fungi.

\section{Chemical structure of polymyxins}

Polymyxins are cyclic polycationic decapeptides that contain a heptapeptide ring and have a high rate of 2,4diaminobutyric (Dab) acid and a fatty acid bound to the peptide by an amide bond. The ring is formed by the $\alpha$ amine and carboxyl groups of the Dab residue in position 
with an additional peptide chain attached through the gamino group of this residue.

\section{Mechanism of action}

Polymyxins are amphipathic antibiotics and act primarily upon the external and cytoplasmic membranes, with similar action to that of simple cationic detergents. ${ }^{3}$

The mechanism of action of Polymyxins is thought to be based on surfactant activity, which disrupts the bacterial outer and cytoplasmic membranes.

They bind to cell envelope components such as phospholipids and liposaccharides (LPS), displacing, in a competitive fashion, the $\mathrm{Ca}$ and $\mathrm{Mg}$ ions that act as membrane stabilizers, leading to membrane rupture and to the loss of cellular contents, thus killing the bacterium. This action seems to be inhibited in the presence of these bivalent cations.

The binding and inactivation capacity of the liposaccharide (LPS), a molecule that is related to the development of sepsis and septic shock, has prompted research into its capacity in treating these syndromes, inhibiting or reducing the inflammatory stimulus induced by LPS. In this regard, devices for LPS removal and inflammatory mediators, such as dialysis filters impregnated with polymyxin $B$, have been used and achieved some success.

The Polymyxins act initially by binding to lipid $A$, the membrane-anchoring component of lipopolysaccharide (LPS), which decorates the outer membrane of Gramnegative bacteria. The anionic nature of LPS facilitates electrostatic interaction with the pentacationic Polymyxins. This initial interaction then leads to disruption of bacterial outer membrane permeability barrier through destabilization of the LPS layer and hydrophobic insertion of the fatty acyl chain of Polymyxin into the lipid domain of lipid A.

Subsequently, cytoplasmic membrane disruption and potential additional intracellular interactions lead to cell death.

\section{Spectrum of activity}

Polymyxin B has no activity against Gram-positive bacteria and anaerobes, but is active against a variety of Gramnegative bacilli, including most clinically relevant Enterobacteriaceae and non-fermentative species. Its spectrum of activity is nearly identical to colistin.

P. aeruginosa and Acinetobacter spp. are intrinsically susceptible, including most of the isolates that are resistant to all the other classes of antibiotics. Stenotrophomonas maltophilia is usually susceptible although some strains are resistant.

Burkholderia cepacia complex and Burkholderia pseudomallei are resistant to polymyxin B. Among Enterobacteriaceae, Escherichia coli, Enterobacter spp., Citrobacter spp., Salmonella spp., Shigella spp. and Klebsiella spp. are usually susceptible. Proteus spp.,
Providencia spp., Morganella morganii and Serratia marcescens are resistant.

Polymyxin B is active against some species of Aeromonas, but Aeromonas jandaei is resistant and Aeromonas hydrophila has inducible resistance.

The pathogenic Neisseria spp. (including meningococci and gonococci), Moraxella catarrhalis, Helicobacter pylori, Vibrio spp. and Brucella spp. are intrinsically resistant. Campylobacter spp. vary in their susceptibility to polymyxin B and the susceptibility of Bartonella spp. is borderline.

\section{Dosing}

Polymyxin B is used at the dose of 1.5 to $2.5 \mathrm{mg} / \mathrm{kg} /$ day (1.0 mg of polymyxin B sulfate $=10,000 \mathrm{IU}$ ) in patients with normal renal function (creatinine clearance $>80 \mathrm{~mL} / \mathrm{min}$ ). In patients with abnormal renal function, it is recommended that a dose of $2.5 \mathrm{mg} / \mathrm{kg}$ be given on the first day, adjusting the subsequent doses according to creatinine clearance $(\mathrm{CrCl})$. Between 30 and $80 \mathrm{~mL} / \mathrm{min}$, 1.0 to $1.5 \mathrm{mg} / \mathrm{kg} / \mathrm{d}$ should be administered; with $\mathrm{CrCl}<30$ $\mathrm{mL} / \mathrm{min}$, the dose ranges from 1.0 to $1.5 \mathrm{mg} / \mathrm{kg}$ every 2 or 3 days; and in anuric patients, administration of $1.0 \mathrm{mg} / \mathrm{kg}$ must be made every 5 to 7 days.

\section{Nephrotoxicity}

The development of renal injury, especially acute renal failure (ARF), is the major limiting factor for the use of this class of antibiotics. There is an increase in the levels of serum urea and creatinine and a decrease in $\mathrm{CrCl}$, in addition to hematuria, proteinuria, cylindruria and oliguria.

The mechanism of renal injury seems to be related to the contents of d-aminobutyric acid and fatty acid of the molecule, and resembles the effect of the antibiotic on the outer bacterial membrane. Membrane permeability increases, making the flow of cations, anions and water easier and causing edema and cell lysis. This action is seemingly dependent upon the concentration and length of exposure to the antibiotic.

Identifying ways to minimize nephrotoxicity associated with polymyxin B use is critical to advance clinical practice. Previous studies have evaluated the prevalence rates of nephrotoxicity, described time to the development of acute kidney injury (AKI), examined the impact of different polymyxin $B$ dosing frequencies on the nephrotoxicity and identified independent predictors of AKI.

Over the last decade, Carbapenem-resistant Gramnegative rods (CRGNRs) have emerged as important health care-associated pathogens that are associated with significant morbidity and mortality. Due to broad antimicrobial resistance among CRGNRs, clinicians have been increasingly forced to rely upon Polymyxins for the treatment of infections caused by these organisms. 
The bactericidal activity of Polymyxin B is concentration dependent and appears to be best correlated with the area under the concentration-time curve over $24 \mathrm{~h}$ in steady state divided by the MIC (AUC/MIC).

Polymyxin B sulfate (PMB), for which early use in the 1970s was discontinued because of its toxicity, especially nephrotoxicity, has reemerged in clinical practice over the last 10 years. The current reuse of PMB is the result of the growing number of infections caused by the highly mutational and adaptive resistance of Gram-negative bacteria.

PMB induces nephrotoxicity characterized by acute tubular necrosis (ATN) and elevated serum creatinine concentration in $37 \%$ of patients.

Polymyxin (PMB) remains the last-line treatment option for life-threatening infections caused by Carbapenemresistant Enterobacteriaceae (CRE), multidrug-resistant (MDR) Pseudomonas aeruginosa, and Acinetobacter baumannii. Nephrotoxicity is the most clinically concerning adverse effect associated with PMB.

Its use has been advocated recently provided other nephrotoxic drugs are not used along with cautious use in geriatric patients. $^{4}$

Attempts to overcome resistance by escalating the dose are hindered by concerns of toxicity. Since the acquired resistance to Polymyxins is still relatively low, studies are urgently needed to delineate their nephrotoxicity potential to guide optimal clinical dosing.

Several reports published in the 1960s and 1970s showed that parenteral administration of Polymyxin was associated with considerable nephrotoxicity. However, since their recent revival, published experiences of systemic Polymyxin use suggested that the incidence of nephrotoxicity was less common and less severe than those reported 40 years ago.

As a consequence of the increasing rates of multidrug resistance in gram-negative bacteria, the Polymyxins have increasingly become the last viable therapeutic option for MDR pseudomonas infections, despite very limited pharmacokinetic and pharmacodynamics data.

Independent risk factors for the onset of polymyxin Bassociated nephrotoxicity identified in few of the studies included daily dose by actual body weight, concurrent use of vancomycin and contrast media. ${ }^{5}$ High doses, age and increased weight were also contributors for the risk. ${ }^{6}$

As we are faced with the possibility of returning to the pre antibiotic era, the Polymyxins are the agents of our last line of defense. It is therefore critical that they be used judiciously and optimally. If the pharmacodynamics of these agents are thoroughly understood, dosing regimens may be designed rationally to optimize patient outcomes and to minimize the emergence of resistance to these agents.
Reported rates of nephrotoxicity associated with the systemic use of polymyxins have varied widely. The reported rates of nephrotoxicity with polymyxins range from 5 to $60 \%$ according to recent studies using standardized criteria to evaluate nephrotoxicity.

There is a need for discovery and development for novel biomarkers which can detect renal damage earlier and aid in preventive measures.

With polymyxin $B$ being an essential antibiotic for MDR and XDR Gram-negative infections, polymyxin B antibiotic stewardship is strongly advised. Infection control and prevention measures should always supplement antibiotic use.

\section{Resistance}

Resistance to these agents, although uncommon, has been shown to occur by mutation or adaptation through a change in the bacterium's outer membrane preventing the drug from reaching the inner cytoplasmic membrane.

Recently, the rise in infections caused by multidrugresistant (MDR) Gram-negative bacteria, especially the resistance to carbapenems most importantly observed in Pseudomonas aeruginosa, Acinetobacter baumannii, Klebsiella pneumoniae, and Escherichia coli, has led to the resuscitation of polymyxins (polymyxin B and colistin) worldwide as a last-resort treatment option. ${ }^{7}$

There has been a report suggesting various mechanisms of resistance to Polymyxin B which includes: PhoP-PhoQ system, species-specific resistance mechanisms and presence of OM proteins in bacteria. ${ }^{8}$

There are also reports of increases in infections caused by naturally polymyxin-resistant bacteria, such as Proteus, Providencia, Morganella, and Serratia.

\section{Aim}

This study aimed to assess the incidence of nephropathy in patients associated with the use of polymyxin B.

\section{Objectives}

- To assess the incidence of nephrotoxicity in patients associated with the use of polymyxin $B$.

- To evaluate the effect of dosing frequency on polymyxin $b$ associated nephrotoxicity.

- To identify the risk factors for acute kidney injury in patients treated with polymyxin B.

- To evaluate the relationship between polymyxin $b$ use and clinical outcomes.

\section{METHODS}

\section{Study selection}

The full text of all potentially eligible articles was obtained. We reviewed the full text articles for eligibility and the duplicate study was excluded. Any disagreements were 
resolved through discussion or after consultation with the senior authors/reviewers, when necessary.

\section{Study sources}

The following data were searched: Medline (PubMed); Cochrane, PubMed, Antimicrobial agents and chemotherapy, Journal of pharmacy practice and WHO regional medical databases. Language was restricted to English.

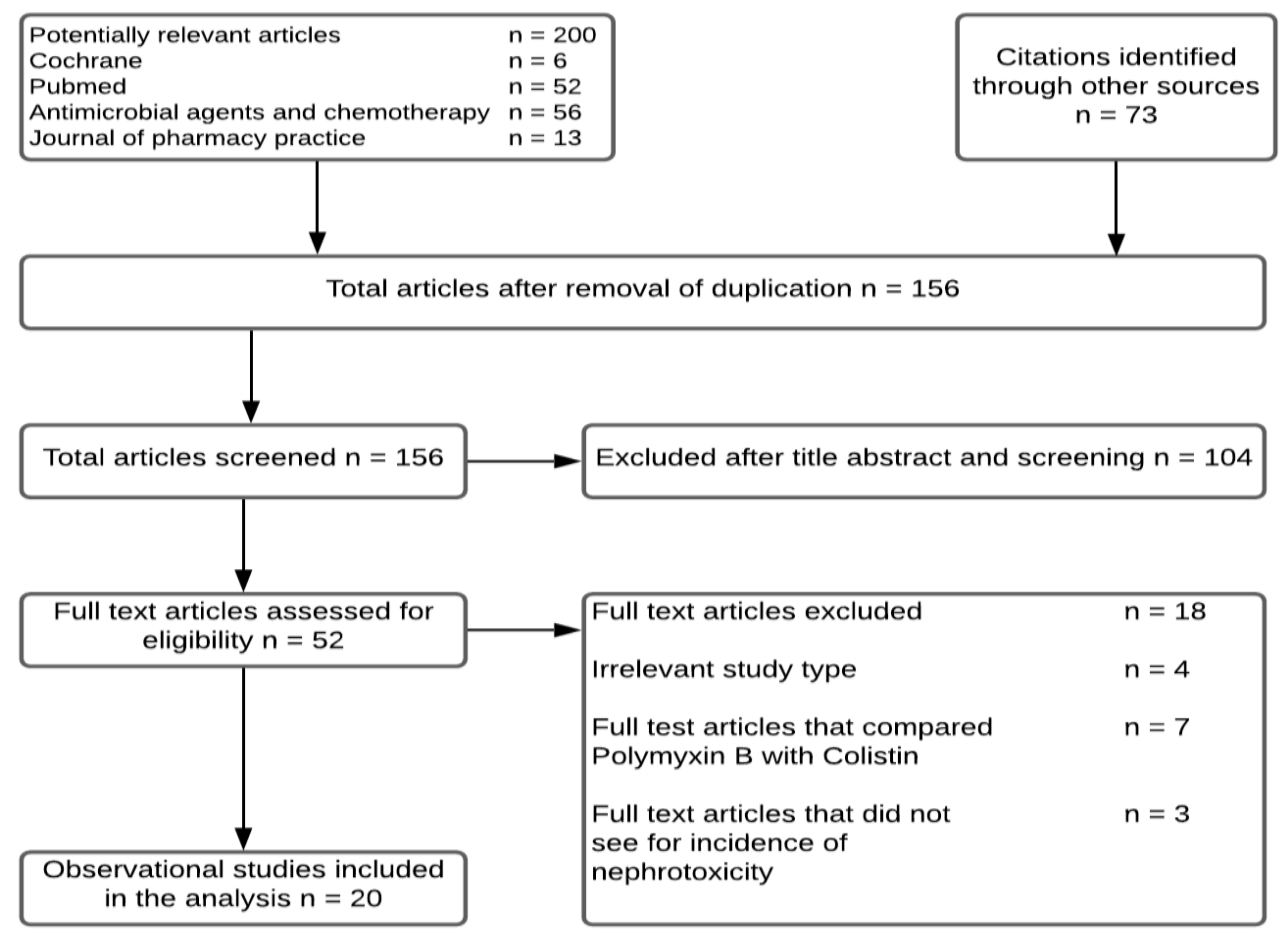

Figure 1: Flow chart for selection of articles included in the study

\section{DISCUSSION}

With increasing variety of antibiotics resistance to gramnegative microorganisms and also some other new effective agents, there has been renewed use of old antimicrobial such as Polymyxin B.

Polymyxin B is an antibiotic which is effective against gram negative and also to some rate of extent it is effective against gram positive bacteria. Nephrotoxicity is a dose limiting adverse effect of Polymyxin. The higher the dose there will be more chances for nephrotoxicity, but currently there is limited data available on the onset of Nephrotoxicity.

A major limitation in the interpretation of polymyxininduced nephrotoxicity and neurotoxicity is the intensive care unit setting.

Dose of Polymyxin is based on body weight. The loading dose is 10 lakh units and the maintenance dose is 5 lakh units. The dose of Polymyxin B varies depending on patient condition and disease severity and also based on mortality and morbidity rate.

Nephrotoxicity to Polymyxin B is related to dosage and duration of therapy. The results play a very important role for implications on possible intervention design to prevent the occurrence of Nephropathy associated with Polymyxin B.

Kidney function needs to be monitored during Polymyxin therapy, especially serum creatinine level which is a major marker for kidney injury.

Kidney damage results from the accumulation of polymyxin $B$ in the organ owing to high reabsorption rates of this drug, notably by the proximal tubular cells in the renal cortex. Additionally, it has been shown that tubular reabsorption of Polymyxin $B$ seems to be a saturable non-passive mechanism. A protective effect of dose on mortality regardless of the development of AKI has previously been found in retrospective study.

We reviewed more than 15 studies from which only $8 \%$ study suggested the rate of Polymyxin $B$ inducing nephrotoxicity and $17 \%$ article shows the effect of Polymyxin B on the kidney by using pharmacodynamics and monitoring creatinine clearance.

While some studies suggested high doses as a risk factor for $\mathrm{AKI}$, one study showed lower in-hospital mortality with a dose $\geq 200$ mg per day. ${ }^{9}$

Earlier onset and increased risk of nephrotoxicity was observed in patients with BMI $\geq 25 \mathrm{~kg} / \mathrm{m} 2$ including overweight, obese and volume - overloaded patients. The 
median duration of polymyxin B therapy was 11 days and the median time to the first sign of AKI was 4 days (range 114 days). ${ }^{10}$ In comparison, Akajagbor et al., reported a mean time to peak serum creatinine of 7.3 days with a mean treatment duration of 12.5 days.

Independent risk factors for the onset of polymyxin Bassociated nephrotoxicity identified in few of the studies included daily dose by actual body weight, concurrent use of vancomycin and contrast media. Concomitant use of aminoglycosides also increased the risk for AKI. ${ }^{11}$

The already reported experience regarding the toxicity of polymyxins in the old literature has led to more correct use of these antibiotics by physicians nowadays. A retrospective study about the efficacy and nephrotoxicity rates has shown that treating multi-resistant gram negative infections with Polymyxin B had low nephrotoxicity rates. ${ }^{12}$

In addition, the avoidance of co-administration of potential nephrotoxic and/or neurotoxic agents with polymyxins, as well as the development of critical care supplies, may also explain the observed differences.

Therapeutic drug monitoring (TDM) could be used as an aid to clinical research as well as practical application, especially in the cases where higher doses were adopted.

\section{CONCLUSION}

The incidence of nephrotoxicity associated with the use of Polymyxin B ranged between $5 \%$ to $55 \%$.

Four cellular mechanisms happen which cause nephrotoxicity, namely: oxidative stress, apoptosis, cell cycle arrest and autophagy. ${ }^{13}$

The frequency of dosing showed reduced toxicity incidence with twice daily dosing compared to once daily dosing.

A few measures can be taken to prevent drug induced nephrotoxicity:

- Dosage adjustment based on Cockcroft-Gault formula (in adults) or Schwartz formula (in children).

- Considering functioning level of kidney and performing baseline renal function

- Avoiding nephrotoxic combinations

- Rectifying the risk factors

- Usage of non-nephrotoxic drugs at all possible times

- Establishing adequate hydration ${ }^{14}$

The risk factors for incidence were identified which includes:

- Obesity

- Volume overload

- Concurrent use of vancomycin
Clusterin, osteopontin, proteinuria, albumin, Cystatin C Retinol binding protein are some of the biomarkers that can be used to detect early damage to kidneys. ${ }^{15}$

Further studies for fully understanding the pharmacokinetics and pharmacodynamics of Polymyxin $B$ is therefore required to use it optimally for designing rational dosage regimens which in turn can lead to better patient outcomes and reduced resistance rates. ${ }^{16}$

To prevent resistance, antimicrobial stewardship programs can be initiated and carried out on a larger scale. Therapeutic drug monitoring can also be conducted to know more about the drug characteristics.

\section{REFERENCES}

1. Bahlis LF, Diogo LP, Lemons D, Klaus D. Risk factors for acute kidney injury in patients treated with polymyxin $B$ at a Tertiary Care Medical Center. Brazilian Journal of Nephrology. 2015 Dec;37(4):446-50. DOI: 10.5935/0101-2800.20150071. PMID: 26648493.

2. Gallardo-Godoy A, Hansford KA, Muldoon C, Becker B, Elliott AG, Huang JX, Pelingon R, Butler MS, Blaskovich MA, Cooper MA. Structure-function studies of polymyxin B lipononapeptides. Molecules. 2019 Jan;24(3):553. DOI: 10.3390/molecules 24030553. PMID: 30717415.

3. Mendes CA, Burdmann EA. Polymyxins-a review focusing on their nephrotoxicity. Rev Assoc Med Bras. 2010;56(6):752-8. DOI: 10.1590/s010442302009000600023. PMID: 20191233.

4. Nandha R, Sekhri K, Mandal AK. To study the clinical efficacy and nephrotoxicity along with the risk factors for acute kidney injury associated with parenteral polymyxin B. Indian journal of critical care medicine: peer-reviewed, official publication of Indian Society of Critical Care Medicine. 2013 Sep;17(5):283. DOI: 10.4103/0972-5229.120319. PMID: 24339639.

5. Dubrovskaya Y, Prasad N, Lee Y, Esaian D, Figueroa DA, Tam VH. Risk factors for nephrotoxicity onset associated with polymyxin B therapy. Journal of Antimicrobial Chemotherapy. 2015 Jun 1;70(6):19037. DOI: 10.1093/jac/dkv014. PMID: 25652747.

6. Rigatto $\mathrm{MH}$, Behle TF, Falci DR, Freitas $\mathrm{T}$, Lopes NT, Nunes M, Costa LW, Zavascki AP. Risk factors for acute kidney injury $(\mathrm{AKI})$ in patients treated with polymyxin $B$ and influence of AKI on mortality: a multicentre prospective cohort study. Journal of Antimicrobial Chemotherapy. 2015 May 1;70(5):1552-7. DOI: 10.1093/jac/dku561. PMID: 25604744.

7. Falagas ME, Kasiakou SK. Toxicity of polymyxins: a systematic review of the evidence from old and recent studies. Critical care. 2006 Feb;10(1):1-3. DOI: 10.1186/cc3995. PMID: 16507149

8. $Y u$ Z, Qin W, Lin J, Fang S, Qiu J. Antibacterial mechanisms of polymyxin and bacterial resistance. 
Biomed Res Int. 2015 Oct;2015(679109). DOI: 10.1155/2015/679109. PMID: 25664322.

9. Elias LS, Konzen D, Krebs JM, Zavascki AP. The impact of polymyxin B dosage on in-hospital mortality of patients treated with this antibiotic. Journal of antimicrobial chemotherapy. 2010 Oct 1;65(10):22317. DOI: 10.1093/jac/dkq285. PMID: 20685752.

10. Zhang B, Zuo W, Gao D, Xu X, Yang Y, Wu B, Zhang Y. $A$ retrospective study of patients receiving polymyxin $\mathrm{B}$ intravenous treatment in PUMCH: real clinical practice. (Preprint from Research Square, 14 Feb 2020). DOI: $10.21203 /$ rs.2.23536/v1.

11. Nelson BC, Eiras DP, Gomez-Simmonds A, Loo AS, Satlin MJ, Jenkins SG, Whittier S, Calfee DP, Furuya EY, Kubin CJ. Clinical outcomes associated with polymyxin $B$ dose in patients with bloodstream infections due to carbapenem-resistant Gram-negative rods. Antimicrobial agents and chemotherapy. 2015 Nov 1;59(11):7000-6. DOI: 10.1128/AAC.00844-15. PMID: 26324272.

12. Ouderkirk JP, Nord JA, Turett GS, Kislak JW. Polymyxin $B$ nephrotoxicity and efficacy against nosocomial infections caused by multiresistant gram-negative bacteria. Antimicrobial agents and chemotherapy. 2003 Aug 1;47(8):2659-62. DOI: 10.1128/AAC.47.8.2659-2662.2003. PMID: 12878536.

13. Avedissian SN, Liu J, Rhodes NJ, Lee A, Pais GM, Hauser AR, Scheetz $\mathrm{MH}$. A review of the clinical pharmacokinetics of polymyxin B. Antibiotics. 2019 Mar;8(1):31-39. DOI: 10.3390/antibiotics801003. PMID: 30909507.

14. Dhodi DK, Bhagat SB, Pathak D, Patel SB. Drug-induced nephrotoxicity. Int J Basic Clin Pharmacol. 2014 Jul;3(4):591-7. DOI:

$10.5455 / 2319$ 2003.ijbcp20140826.

15. Kim SY, Moon A. Drug-induced nephrotoxicity and its biomarkers. Biomolecules \& therapeutics. 2012 May;20(3):268.

DOI: 10.4062/biomolther.2012.20.3.268. PMID: 24130922.

16. Tam VH, Schilling AN, Vo G, Kabbara S, Kwa AL, Wiederhold NP, Lewis RE. Pharmacodynamics of polymyxin $B$ against Pseudomonas aeruginosa. Antimicrobial agents and chemotherapy. 2005 Sep 1;49(9):3624-30. DOI: 10.1128/AAC.49.9.36243630.2005. PMID: 16127031.

Source of Support: The author(s) received no financial support for the research, authorship, and/or publication of this article.

Conflict of Interest: The author(s) declared no potential conflicts of interest with respect to the research, authorship, and/or publication of this article.

For any question relates to this article, please reach us at: globalresearchonline@rediffmail.com New manuscripts for publication can be submitted at: submit@globalresearchonline.net and submit_ijpsrr@rediffmail.com 